\title{
Outcomes of androgen replacement therapy in adult male hypogonadism: recommendations from the Italian society of endocrinology
}

\author{
A. M. Isidori • G. Balercia • A. E. Calogero • \\ G. Corona $\cdot$ A. Ferlin $\cdot$ S. Francavilla $\cdot$ \\ D. Santi $\cdot$ M. Maggi
}

Received: 31 May 2014/ Accepted: 6 August 2014/Published online: 11 November 2014

(C) Italian Society of Endocrinology (SIE) 2014

\begin{abstract}
Objective We developed clinical practice guidelines to assess the individual risk-benefit profile of androgen replacement therapy in adult male hypogonadism (HG), defined by the presence of specific signs and symptoms and serum testosterone $(T)$ below $12 \mathrm{nmol} / \mathrm{L}$.

Participants The task force consisted of eight clinicians experienced in treating HG, selected by the Italian Society of Endocrinology (SIE). The authors received no corporate funding or remuneration.

Consensus process Consensus was guided by a systematic review of controlled trials conducted on men with a mean $T<12 \mathrm{nmol} / \mathrm{L}$ and by interactive discussions. The guidelines were reviewed and sequentially approved by the SIE Guidelines Commission and Executive Committee. Conclusions We recommend $T$ supplementation (TS) for adult men with severely reduced $T$ levels $(T<8 \mathrm{nmol} / \mathrm{L})$
\end{abstract}

A. M. Isidori

Department of Experimental Medicine,

Sapienza University of Rome, Rome, Italy

G. Balercia

Department of Clinical and Molecular Sciences,

Polytechnic University of Marche, Ancona, Italy

\section{A. E. Calogero}

Department of Medical and Pediatric Sciences,

University of Catania, Catania, Italy

G. Corona

Medical Department Endocrinology Unit,

Maggiore-Bellaria Hospital, Bologna, Italy

A. Ferlin

Department of Medicine, University of Padua,

Padua, Italy to improve body composition and sexual function. We suggest that TS be offered to subjects with $T<12 \mathrm{nmol} / \mathrm{L}$ to improve glycaemic control, lipid profile, sexual function, bone mineral density, muscle mass and depressive symptoms, once major contraindications have been ruled out. We suggest that lifestyle changes and other available interventions (e.g. for erectile dysfunction) be suggested prior to TS. We suggest that TS should be combined with currently available treatments for individuals at high risk for complications, such as those with osteoporosis and/or metabolic disorders. We recommend against using TS to improve cardiac outcome and limited mobility. We recommend against using TS in men with prostate cancer, unstable cardiovascular conditions or elevated haematocrit. The task force places a high value on the timely treatment of younger and middle-aged subjects to prevent the longterm consequences of hypoandrogenism.

\footnotetext{
S. Francavilla

Department of Life, Health and Environment Sciences, Andrology, and Epidemiology, University of L'Aquila, L'Aquila, Italy

D. Santi

Department of Biosciences, Metabolism and Neural Sciences, University of Modena and Reggio Emilia, Modena, Italy

M. Maggi $(\bowtie)$

Sexual Medicine and Andrology Unit,

Department of Experimental and Clinical Biomedical Sciences, University of Florence, Viale Pieraccini 6, 50139 Firenze, Italy

e-mail: m.maggi@dfc.unifi.it
} 


\section{Methodology and definitions}

The recommendations were based exclusively on evidence from randomised controlled trials (RCTs) of hypogonadal (HG) subjects, defined by a mean serum testosterone (T) level below $12 \mathrm{nmol} / \mathrm{L}$. Data from undefined and mixed populations (eugonadal/HG) or from non-RCTs were analysed separately and taken into account for the formulation of values and remarks only.

A number of problems were encountered in the reviewing process. These pertained to: (a) poor biochemical and clinical definition of $\mathrm{HG}$ in the inclusion criteria; (b) variability in the age of enrolled subjects, with the majority of studies performed in middle-aged subjects (below 75 years); (c) variability in $\mathrm{T}$ formulation and dose, ranging from suboptimal to supra-physiological regimens; (d) inconsistencies in the definition of comorbidities (especially for metabolic and cardiovascular disorders); (e) extreme variability in the duration of treatment (from 4 weeks to 36 months); (f) heterogeneity in the methods for assessing the primary outcomes; (g) no study primarily designed to assess cardiovascular or prostate outcomes; (h) study duration insufficient to address cardiovascular and prostate safety; (i) unstated or underpowered sample sizes for either the primary or secondary outcomes for most studies.

The task force used the following coding system: (1) indicates a strong recommendation and is associated with the phrase "we recommend"; (2) denotes a weak recommendation and is associated with the phrase "we suggest." Evidence grading: ØООО denotes very low quality evidence; ØØОО, low quality; ØØØО, moderate quality; and $\varnothing \varnothing \varnothing \varnothing$, high quality.

The term $T$ replacement therapy has been shortened to TS. For all recommendations, when not otherwise specified, HG was defined as mean circulating $T$ levels below $12 \mathrm{nmol} / \mathrm{L}$ and the presence of specific signs and symptoms, reviewed as potential outcomes: reduced muscle mass and increased adiposity, altered glucose metabolism, reduced bone density, low libido, erectile dysfunction, depression, HIV-related muscle wasting, impaired exercise capacity and anaemia. The formulated recommendations do not apply to men with mean $T$ values above $12 \mathrm{nmol} / \mathrm{L}$.

\section{Metabolic outcomes}

1. We suggest TS for HG subjects with metabolic syndrome (MetS) and type 2 diabetes mellitus (T2DM) to improve glycaemic control (2 ØØОО).

2. We suggest TS for HG subjects to improve lipid profile (2 ØØОO).
3. We recommend TS to reduce fat mass in HG subjects (1 ØØØО), and we suggest it for subjects with either MetS or T2DM (2 ØØOO).

4. We suggest TS to increase lean mass in HG subjects (2 ØØ○) and in those with MetS and T2DM (2 ØØOO).

5. We suggest TS to reduce waist circumference in $\mathrm{HG}$ subjects with MetS and T2DM (2 ØØOO).

\section{Evidence}

In HG subjects with MetS and/or T2DM, a limited number of studies show a tendency towards improved glycaemic control after TS [1]. However, this trend is not evident in the general HG population [2-4] and is less evident in MetS. Conversely, lipid profile, which is tested in a higher number of studies, seems to improve after TS in all HG patients [5-8], but this is not so evident in HG subjects with MetS and/or T2DM [9-11]. Body mass index (BMI) is not affected by TS in HG subjects with or without metabolic disorders $[3,9,10]$. A clear-cut decrease in fat body mass during TS is reported in almost all studies performed in unselected HG subjects [12] and in the fewer studies performed in HG patients with MetS and/or T2DM [9-11, 13, 14]. Similarly, lean mass increases in all HG patients receiving TS, although the trend is less evident in patients with dysmetabolism.

\section{Value}

We place an important value on changes to body composition and metabolic profile in men with unequivocally reduced $\mathrm{T}$ levels.

\section{Remarks}

Although the efficacy of TS in improving body composition has been consistently replicated, the task force remarks that TS should not be administered as an alternative to appropriate lifestyle changes [15], which should be pursued prior to or, in selected cases, alongside TS.

\section{Bone outcomes}

6. We suggest TS in men with reduced $\mathrm{T}$ levels to improve, or avoid worsening of, lumbar (2 ØØОО) and femoral (2 ØООО) bone mineral density.

\section{Evidence}

$T$ has a fundamental role in bone maturation: at the end of puberty to reach peak bone mass, and during adult life to 
maintain it [16]. HG men have significantly lower bone mineral densities (BMD) than eugonadal age-matched men [16]. The prevalence of HG in osteoporotic men, however, has not been properly assessed. Studies estimate HG in up to $20 \%$ of men with vertebral fractures and $50 \%$ of hip fractures [17]. Some non-placebo-controlled trials and/or trials in strictly selected non-HG men without other major comorbidities are available [18-22]. Taken together, these studies suggest a positive effect of TS on lumbar spine but not femoral neck BMD. Only two RCTs [23, 24] have been conducted in elderly and clearly HG men $(T<12 \mathrm{nmol} / \mathrm{L})$, for a total of 41 subjects [23, 24], treated with intramuscular $T$ injection. These studies show a significant effect of TS on femoral BMD, as compared to placebo $(+2.7 \%)$ [23, 24], and a more pronounced effect $(+10.2 \%)$ on lumbar BMD, after 36 months [23]. Another controlled study, performed on 99 HG men with a history of fracture or $T$-score $<-2.0$ and frailty, shows a positive effect of transdermal TS on lumbar BMD [20]. One non-placebo-controlled RCT study performed on $40 \mathrm{HG}$ men with sexual symptoms and MetS or T2DM shows a positive relationship between serum $T$ and BMD increments at both the lumbar and femoral sites after 36 months of $T$ undecanoate treatment [18].

\section{Values}

Evidence supporting TS to increase lumbar BMD in HG men is of low quality. The evidence for femoral BMD is of very low quality. The task force places a high value on the long-term (36 months) composite benefits of TS on the musculoskeletal system, especially when $T$ levels are very low $(<8 \mathrm{nmol} / \mathrm{L})$.

\section{Remarks}

Although there is no doubt that $T$ is a key hormone for skeletal health, a careful diagnosis should be made to characterise HG, excluding other causes of low BMD and understanding the relative role of other comorbidities, especially in men with only a marginal reduction in $T$ levels. No studies report effects on fracture rates after TS, nor is there any evidence for the effects of co-treatment with agents with proven antifracture efficacy (e.g. bisphosphonates) and/or vitamin D/calcium supplementation. Similarly, there is no clear evidence of better results with $T$ injections than with transdermal administration. For this reason, the task force suggests adding other pharmacological agents to treat osteoporosis if the fracture risk is high. Men with a modest or borderline risk of fracture can be treated with TS alone. In general, the decision on if and when to add vitamin $\mathrm{D}$, calcium and/or another specific treatment and which $T$ formulation to use should be based on an assessment of the risks and benefits for the patient concerned.

\section{Cardiovascular outcomes}

7. We suggest that clinicians exercise caution in giving $T$ to older men with known cardiovascular disease (CVD), due to an unclear benefit/risk ratio (2 ØØОО).

8. In patients deserving treatment, we suggest offering long-acting injectable $T$ esters or transdermal preparations rather than short-acting $T$ esters, to minimise the risk of increased haematocrit, a potential cardiovascular risk factor (2 ØОOО).

9. We suggest considering TS for middle-aged HG men with metabolic disorders without known CVD, to decrease the risk of future cardiovascular events (2 ØОOО).

10. We recommend against $\mathrm{TS}$ to improve cardiac dysfunction in HG men (1 ØОOO).

\section{Evidence}

The effects of TS on cardiovascular function, events and mortality of HG patients are poorly known [25]. Some studies show beneficial effects on surrogate markers of cardiovascular health $[8,14,26]$. The few RCTs and subsequent meta-analyses [27-29] suggest that TS in men is not associated with a consistent CV effect. Most trials rely on too few adverse events to reach reliable conclusions. A recent meta-analysis of RCTs [30] identified 27 trials including 2,994 middle-aged or elderly men (1733 T-treated) with a baseline $T$ level ranging between 7.0 and $20.0 \mathrm{nmol} / \mathrm{L}$, reporting $180 \mathrm{CV}$-related events, including $\mathrm{CV}$-related deaths. $T$ treatment increased the risk of $\mathrm{CV}$ related events (OR 1.54, $95 \%$ CI 1.09-2.18), including CV-related deaths (OR 1.42; $95 \%$ CI 0.70-2.89), independently of baseline $T$ level [30]. However, a more recent meta-analysis [31] on a much larger population $(5,508$ men, 3,040 treated with $T$ ) confutes these findings, showing no effect on major cardiovascular events (MACE) and mortality, and a somewhat CV-protective effect in a subgroup of HG men with metabolic diseases (OR $0.1995 \%$ CI $0.04 ; 0.85)$. The major limitation is that none of these RCTs had CV function or events as the primary or secondary outcome, and all studies were underpowered to address this issue. In addition, reporting of adverse events, bias linked to trial funding $[30,32]$ and study duration too short to define CV safety all undermine the validity of the currently available data. A qualitative review of the trials seems to suggest that long-acting injectable $T$ esters or transdermal preparations should be preferred to reduce the risk of excessive haematocrit increase during TS [28, 33], although a formal quantitative appraisal is still lacking [30]. 
Values

The recommendation to offer TS to elderly HG men places a relatively low value on avoiding the burden of TS and its unclear cardiovascular effects.

\section{Remarks}

The risks of treating elderly HG men with TS appear to be related to age, history of previous $\mathrm{CV}$ events and rise in haematocrit. In younger and middle-aged HG subjects [34], these risk factors seem, on the whole, to play a minor role compared to the potential benefits of TS in reducing several other cardiovascular risk factors.

\section{Sexual function}

11. We recommend TS for men with symptoms of low libido who have markedly reduced $T$ levels ( $T<8 \mathrm{nmol} / \mathrm{L})$ to improve libido (1 ØØØO); whilst we suggest TS in men with $T$ levels between 8 and $12 \mathrm{nmol} / \mathrm{L}(2 \varnothing \varnothing \varnothing \mathrm{O})$.

12. We recommend that clinicians offer TS to men with erectile dysfunction (ED) who have markedly reduced $T$ levels ( $T<8 \mathrm{nmol} / \mathrm{L}$ ), to improve erection (1 ØØØO).

13. In men with $T$ levels between 8 and $12 \mathrm{nmol} / \mathrm{L}$ and ED, we suggest considering TS after having tried the established therapies to recover sexual function (2 $\varnothing \varnothing \varnothing О)$. In non-responders to phosphodiesterase 5 (PDE5) inhibitors who retain persistently low $T$ levels, we suggest offering $T$ therapy to improve erectile function (2 ØØОО).

14. We suggest that clinicians offer TS to men with low $T$ levels $(T<12 \mathrm{nmol} / \mathrm{L})$ to improve orgasmic function (2 ØОOO).

\section{Evidence}

Observational data show that sexual complaints, including $\mathrm{ED}$, are the most common symptoms associated with $\mathrm{HG}$ $[35,36]$. Simple correlation, however, does not imply causation. Several non-placebo-controlled trials are available [37-40]. The few RCTs contain a sufficiently large number of treated subjects to draw reliable conclusions [37-39]. The effects of TS on libido have recently been meta-analysed [38]. In 17 RCTs, enrolling 1,111 men, TS determined an improvement in the libido of severely $\mathrm{HG}$ subjects $(T<8 \mathrm{nmol} / \mathrm{L})$, but not in those with milder forms of HG. The effects of TS on sex-related erections were assessed in 1,431 individuals, proving effective only in men with reduced $T$ levels $(<12 \mathrm{nmol} / \mathrm{L})$ [38]. An inverse relationship between mean $T$ levels at enrolment and the effect of TS on overall erectile function was observed. Amongst studies providing International Index of Erectile Function (IIEF) data, TS resulted in a weighted mean improvement of about $40 \%$ (range: 22-79\%), corresponding to a weighted mean delta of $4 \pm 2$ points in the IIEF-5 score (range: 3-8) [39]. Clinical studies document that $T$ not only controls libido and erectile function but is also involved in the regulation of the orgasmic component of male ejaculatory reflex, acting both centrally and peripherally [38]. Ten studies enrolling 677 patients investigated the effect of TS on orgasm, confirming the relationship between baseline mean $T$ levels and the effect size on TS orgasmic function.

Values

There is enough evidence to support the use of TS for men with unequivocally low $T$ levels and sexual complaints. The task force places a high value on the composite benefits of treating young $\mathrm{HG}$ men and a lower value on the treatment of older men, due to the uncertainty of long-term CV safety.

\section{Remarks}

The decision as to whether or when to treat $\mathrm{HG}$ men to improve sexual symptoms remains problematic. ED generally has a multifactorial aetiology; vascular factors are predominant amongst the comorbidities and hamper the beneficial effects of TS. In young HG subjects, TS is often sufficient to improve sexual function. In older men, the low $T$ level may be the consequence rather than the cause of ED [41]. A large number of HG ED patients could be safely treated with PDE5-I monotherapy and TS could be offered as an add-on treatment only for those with persistently low $T$ levels, [39, 41]. The decision to start TS in older HG men depends on the patient's individual benefit/risk ratio.

\section{Other outcomes}

15. We suggest that clinicians offer TS to HG men with depression to improve depressive symptoms (2 ØØОО), in addition to the established therapies for depression.

16. We suggest that clinicians offer TS to $\mathrm{HG}$ men and HIV/AIDS to improve lean mass (2 ØOOO).

17. We suggest against the use of TS to improve exercise capacity in individuals with chronic obstructive pulmonary disease (COPD) and frail men (2 ØOOO) or to improve quality of life (QoL) in subjects with end-stage renal diseases (ESRD) or undergoing glucocorticoid or opioid therapy (2 ØОOО). 
Evidence

\section{Depression}

Depressed mood is a symptom often associated with HG, suggesting that androgen deficiency contributes to mood disorders [1, 42, 43]. It is unclear, however, whether low $T$ levels are correlated with the development of major depressive disorder [42]. The relationship between low $T$ and the incidence of clinical depression is still unclear, especially in elderly men [42]. Few RCTs have evaluated the effect of TS on depressive symptoms (Table 1). Zarrouf et al. [43] evaluated the impact of TS on major depressive disorder according to DSM-IV-defined criteria in seven trials including 355 patients with an age range of 18-70 years. They found a positive effect of TS on Hamilton-D depression rating scale scores in depressed patients with $T<10 \mathrm{nmol} / \mathrm{L}$ and in patients with HIV/AIDS. Similar results are reported in a more up-to-date metaanalysis [44].

\section{HIV/AIDS}

The prevalence of low T levels in HIV-infected men ranges from 20 to $25 \%$ [1, 45, 46]. Low $T$ is closely correlated with weight loss, progression to AIDS, wasting syndrome, depression and loss of muscle mass and exercise capacity [45]. The mechanisms underlying the association between HG and HIV have been reviewed elsewhere [45]. In a recent meta-analysis of the available RCTs in HIV-positive men, including 344 individuals, Corona et al. [1] found that TS improved lean mass over placebo. However, results from these trials were heterogeneous and limited by the small sample size. In particular, when more selective criteria for $\mathrm{HG}$ were applied $(T<12 \mathrm{nmol} / \mathrm{L})$ only two trials were eligible (Table 1).

\section{Other diseases}

Current guidelines [47, 48] suggest that clinicians consider case detection through measuring total $T$ levels in men with certain clinical conditions (including CPOD, ESRD or in those treated with opioid or glucocorticoid therapy). However, the limited evidence from RCTs in these particular populations does not permit any final conclusions to be drawn about the specific role of TS in these subjects or in frailty [1].

\section{Values}

The task force places a relatively high value on the recommendation to offer TS to HG men with major depressive disorders and in those resistant to the use of common antidepressants, and a high value on TS in HIV-infected men with $\mathrm{HG}$ to prevent and/or combat wasting syndrome by improving lean body mass.

\section{Remark}

It should be recognised that evidence for the role of TS in depressed or HIV men is limited, considering both the number of patients enrolled and the follow-up. Further information on the long-term benefits and adverse effects of $T$ use in these subjects is therefore required. The outcomes of TS in other disorders, including CPOD and ESRD, in frail subjects or in those treated with glucocorticoids or opioids are the same as for patients with classic androgen deficiency $[47,48]$.

\section{The prostate}

18. We recommend against limiting TS in HG men with the aim of preventing a new incidence of prostate cancer (PCa) (1 ØØØO).

19. We recommend against using TS in $\mathrm{HG}$ men with $\mathrm{PCa}$ and we recommend against using TS, without further urological investigation, when total PSA is above the normal or in the presence of an abnormal digital rectal examination (palpable nodule) (1 ØØОО).

20. We suggest considering TS in men with signs and symptoms of HG after at least 12 months of clinical and biochemical cure following radical prostatectomy for PCa, but only under strict monitoring (2 ØООО).

21. We suggest against using TS in HG men with an overt urinary tract obstruction due to benign prostatic enlargement (BPE) (2 ØООО).

22. We recommend long-term follow-up in men with markedly low $(<8 \mathrm{nmol} / \mathrm{L})$ or borderline $(8-12 \mathrm{nmol} /$ L) T levels and symptoms of HG who are given TS; this should be stricter in the first year [3, 6 (or 9 according to the clinician's judgement) and 12 months] and at least annually thereafter, with measurement of total (and free) PSA and evaluation of prostate volume by digital rectal examination (1 ØØØО) or trans-rectal ultrasound.

\section{Evidence}

There are no data suggesting that TS is associated with an increased risk of prostate cancer [49]. Observational data on $>1,100$ subjects revealed that about $64 \%$ of HG men treated with $\mathrm{T}$ do not show an increase in PSA. Six RCTs found that TS increases PSA values [6, 23, 50-53], but only in two was the increase significant $[52,53]$. The 
Table 1 Evidence from available RCTs in support of the provided recommendations

\begin{tabular}{|c|c|c|c|c|c|}
\hline $\begin{array}{l}\text { Characteristics of study } \\
\text { population }\end{array}$ & Outcome & $\begin{array}{l}\text { Number of } \\
\text { available } \\
\text { RCTs }\end{array}$ & $\begin{array}{l}\text { Number of } \\
\text { enrolled } \\
\text { subjects }\end{array}$ & $\begin{array}{l}\text { Mean duration } \\
\text { of RCT months } \\
\text { (range) }\end{array}$ & $\begin{array}{l}\text { Summary of } \\
\text { observed } \\
\text { effects }\end{array}$ \\
\hline \multicolumn{6}{|c|}{ Body composition (for additional data see references [1-15]) } \\
\hline MetS or T2DM & Waist circumference & 6 & 701 & $10.7[2.7-24]$ & $\downarrow$ \\
\hline Metabolically unclassified & Waist circumference & 3 & 172 & $6.6[3-11]$ & $\leftrightarrow$ \\
\hline MetS or T2DM & BMI & 8 & 773 & $8.8[2.7-24]$ & $\leftrightarrow$ \\
\hline Metabolically unclassified & BMI & 5 & 307 & $11.2[3-24]$ & $\leftrightarrow$ \\
\hline MetS or T2DM & Body fat & 5 & 379 & $10.7[3-24]$ & $\downarrow$ \\
\hline Metabolically unclassified & Body fat & 10 & 1,513 & $11[3-36]$ & $\downarrow$ \\
\hline MetS or T2DM & Body lean & 4 & 174 & $10.4[2.7-24]$ & $\uparrow$ \\
\hline Metabolically unclassified & Body lean & 9 & 1,491 & $10.8[3-36]$ & $\uparrow$ \\
\hline \multicolumn{6}{|c|}{ Metabolism (for additional data see references [1-15]) } \\
\hline MetS or T2DM & Glycaemia & 7 & 725 & $9.6[2.7-24]$ & $\leftrightarrow$ \\
\hline Metabolically unclassified & Glycaemia & 5 & 324 & $9.4[3-24]$ & $\leftrightarrow$ \\
\hline MetS or T2DM & Glycated haemoglobin & 6 & 555 & $10[2.7-24]$ & $\downarrow$ \\
\hline MetS or T2DM & Total cholesterol & 7 & 725 & $9.6[2.7-24]$ & $\leftrightarrow$ \\
\hline Metabolically unclassified & Total cholesterol & 9 & 490 & $11.7[1-36]$ & $\downarrow$ \\
\hline MetS or T2DM & HDL cholesterol & 7 & 725 & $9.6[2.7-24]$ & $\leftrightarrow$ \\
\hline Metabolically unclassified & HDL cholesterol & 10 & 546 & $13[1-36]$ & $\leftrightarrow$ \\
\hline MetS or T2DM & LDL cholesterol & 5 & 633 & $6.2[2.7-12]$ & $\leftrightarrow$ \\
\hline Metabolically unclassified & LDL cholesterol & 8 & 464 & $15.5[1-36]$ & $\downarrow$ \\
\hline MetS or T2DM & Triglycerides & 7 & 725 & $9.6[2.7-24]$ & $\leftrightarrow$ \\
\hline Metabolically unclassified & Triglycerides & 8 & 380 & $8.7[1-36]$ & $\downarrow$ \\
\hline \multicolumn{6}{|c|}{ Bone density (for additional data see references [16-24]) } \\
\hline Healthy men & Lumbar and femoral bone mineral density & 2 & 41 & $12-36$ & $\begin{array}{l}\uparrow \text { lumbar } \\
\uparrow \text { femoral }\end{array}$ \\
\hline $\begin{array}{l}\text { History of fracture or } \\
T \text {-score }<-2.0 \text { and frailty }\end{array}$ & $\begin{array}{l}\text { Lumbar, femoral and radius bone mineral } \\
\text { density }\end{array}$ & 1 & 53 & 12 & $\begin{array}{l}\uparrow \text { lumbar } \\
\downarrow \text { radius }\end{array}$ \\
\hline \multicolumn{6}{|c|}{ Sexual function (for additional data see references [35-41]) } \\
\hline $\begin{array}{l}\text { Young to middle-aged men with } \\
\text { sexual dysfunction }\end{array}$ & Libido & 17 & 1,111 & 3 months $(1-12)$ & $\begin{array}{l}\uparrow \\
\text { (if } T<8 \\
\text { nM/L) }\end{array}$ \\
\hline $\begin{array}{l}\text { Young to middle-aged men with } \\
\text { sexual dysfunction }\end{array}$ & Sexual related erections & 24 & 1,431 & 3 months $(1-12)$ & $\begin{array}{l}\uparrow \\
\text { (if } T<12 \\
\mathrm{nM} / \mathrm{L} \text { ) }\end{array}$ \\
\hline $\begin{array}{l}\text { Young to middle-aged men with } \\
\text { sexual dysfunction }\end{array}$ & Orgasmic function & 10 & 677 & 3 months $(1-12)$ & $\begin{array}{l}\uparrow \\
(\text { if } \mathrm{T}<12 \\
\mathrm{nM} / \mathrm{L})\end{array}$ \\
\hline \multicolumn{6}{|c|}{ Prostate gland (for additional data see references $[6,9,10,23,49-55]$ ) } \\
\hline $\begin{array}{l}\text { Middle-aged to aged men with } \\
\text { hypogonadism }\end{array}$ & Serum PSA & 6 & 286 & $16.5[3-36]$ & $\leftrightarrow$ \\
\hline $\begin{array}{l}\text { Men with hypogonadism } \\
\text { (unspecified age or wide age } \\
\text { range) }\end{array}$ & Serum PSA & 10 & 461 & 6 [3-12] & $\leftrightarrow$ \\
\hline $\begin{array}{l}\text { Middle-aged men with } \\
\text { hypogonadism }\end{array}$ & $\begin{array}{l}\text { Prostate volume or prostate-related } \\
\text { symptoms (DRE and evaluation of low } \\
\text { urinary tract function) }\end{array}$ & 7 & 256 & 10 [3-36] & $\leftrightarrow$ \\
\hline $\begin{array}{l}\text { Men with hypogonadism and } \\
\text { prostate cancer }\end{array}$ & $\begin{array}{l}\text { Serum PSA, prostate volume or symptoms } \\
\text { (urine flow rate, voiding symptoms, } \\
\text { prostate biopsy) }\end{array}$ & 1 & 21 & 6 & $\leftrightarrow$ \\
\hline
\end{tabular}


Table 1 continued

\begin{tabular}{|c|c|c|c|c|c|}
\hline $\begin{array}{l}\text { Characteristics of study } \\
\text { population }\end{array}$ & Outcome & $\begin{array}{l}\text { Number of } \\
\text { available } \\
\text { RCTs }\end{array}$ & $\begin{array}{l}\text { Number of } \\
\text { enrolled } \\
\text { subjects }\end{array}$ & $\begin{array}{l}\text { Mean duration } \\
\text { of RCT months } \\
\text { (range) }\end{array}$ & $\begin{array}{l}\text { Summary of } \\
\text { observed } \\
\text { effects }\end{array}$ \\
\hline $\begin{array}{l}\text { Men with hypogonadism and } \\
\text { diabetes mellitus type } 2 \text { or } \\
\text { Metabolic syndrome }\end{array}$ & Serum PSA, Prostate volume, IPSS & 3 & 239 & $10[7-12]$ & $\leftrightarrow$ \\
\hline \multicolumn{6}{|c|}{ Other parameters (for additional data see references $[1,42-48]$} \\
\hline Major depression & Improvement of depressive symptoms & 5 & 210 & $7.6(6-12)$ & $\uparrow$ \\
\hline HIV/AIDS & Improvement of lean mass & 2 & 82 & $21(16-26)$ & $\uparrow$ \\
\hline
\end{tabular}

$\leftrightarrow$ no modification or conflicting results after TS; $\uparrow$ increased during TS; $\downarrow$ decreased during TS

majority of patients had a clinical condition of $\mathrm{HG}$ with markedly reduced $\mathrm{T}$ levels $(<8 \mathrm{nmol} / \mathrm{L})$ or $\mathrm{T}$ levels between 8 and $12 \mathrm{nmol} / \mathrm{L}$ and symptoms of $\mathrm{HG}$. A smaller proportion of patients showed comorbidities such as T2DM or MetS [9, 10, 54]. Only $23 \%$ of 250 patients had an increase in prostate volume (assessed by digital rectal examination or prostate ultrasound scan) $[6,23,55]$, whilst in the remaining it was within the normal range.

\section{Values}

The task force places high value on the recommendation that TS, when appropriately given to $\mathrm{HG}$ men, has no carcinogenic effect on an otherwise unaffected prostate gland.

\section{Remarks}

The effects of TS on the prostate gland are not such as to advise limiting its use, given its potential benefits on various cardiovascular, metabolic and sexual outcomes. TS may therefore be considered safe for the prostate gland in all patients without clinically evident $\mathrm{PCa}$, as long as accurate screening is performed beforehand and adequate follow-up is performed.

\section{Adverse effects}

23. We recommend against using $\mathrm{TS}$ in patients with haematocrit higher than the upper limit of the normal

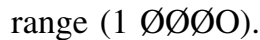

24. We recommend monitoring blood parameters to avoid critical elevation of haematocrit, especially when using intramuscular $\mathrm{T}$ preparations (1 ØØОО).

25. We suggest against using 17-alpha-alkylated derivatives of $T$. The available $T$ preparations do not have negative effects on liver function [48] (2 ØØОО).

26. We recommend against starting $\mathrm{TS}$ in $\mathrm{HG}$ patients who are attempting to father a child (1 ØØØО).
27. We suggest against using TS in men with a known history of obstructive sleep apnoea (2 ØOOO).

\section{Evidence}

TS induces a dose-dependent increase in haematocrit within 1 month [27, 28]. This effect is higher with shortacting than with long-acting, injectable $T$ esters or transdermal preparations [33]. The increase is caused by both erythropoietin and the direct effect of $T$ on bone marrow [56]. Fernandez-Balselles et al. [28] showed that T-treated men had a 3.15-times higher risk of developing erythrocytosis compared to placebo-treated controls, especially with intramuscular $T$ formulations. However, this rarely results in thrombosis, as demonstrated by meta-analyses considering all cardiovascular events [1, 27, 28]. Nevertheless, frequent monitoring is necessary to avoid critically elevated levels, with $54 \%$ considered the cut-off for suspension [48]. Polycythaemia reverts to baseline after $T$ withdrawal. RCTs rarely report increased haematocrit as an adverse event of TS (Table 1). Some studies report a significant haematocrit increase with intramuscular TS [10, 57-59]. However, only a small number of HG patients were considered and haematocrit was not the primary endpoint.

The negative effect of TS on liver function is only associated with the use of alkylated $17 \alpha \mathrm{T}$ formulations, which have already been withdrawn from the market. Long-term studies showing the liver effects of the currently used $\mathrm{T}$ preparations are lacking.

TS down-regulates the hypothalamus-pituitary axis, depressing LH and FSH and further decreasing endogenous $\mathrm{T}$ synthesis, thereby inhibiting sperm production [48]. Androgens have been actively studied for potential use in male hormonal contraception. The negative effect of $\mathrm{T}$ on fertility is transient and fully reversible, with a predictable course [60].

Few studies have considered the effects of TS on obstructive sleep apnoea syndrome (OSAS) in patients with 
HG. One randomised, placebo-controlled study showed that intramuscular $T$ undecanoate acutely worsened sleep hypoxaemia in patients with obesity and OSAS. An openlabel study on five HG men reported worsened OSAS with intramuscular $T$ enanthate [61]. However, Snyder et al. [62] did not find any change in respiratory distress index in 108 HG men treated with transdermal $T$ for 36 months.

Few randomised, placebo-controlled trials indicate which adverse events resulted in patients dropping out of the study. In men treated with transdermal $T$ erythema, pruritus and nasopharyngitis occurred in about $45 \%$ of patients in both the study and control groups [10]. Transdermal approaches might increase the risk of transfer of $T$ to others during close skin contact, although this was found unlikely in a randomised controlled study [63].

\section{Values}

There is enough evidence to recommend avoiding the use of TS in men with elevated haematocrit levels. To prevent this adverse effect, the task force gives a high value to monitoring haematocrit during treatment. Similarly, a high value is given to the negative effect of $T$ treatment in patients requiring fertility. Few RCTs have evaluated TS in men with OSAS.

\section{Remarks}

There is no clear haematocrit threshold above which TS should be avoided or suspended, therefore, continuous monitoring of the individual risk profile (e.g. dehydration, hypercoagulability, smoking) is mandatory during $T$ treatment. At the same time, any wish to start a family should be considered before starting TS. Finally, OSAS is a complex disease and generally has a multifactorial aetiology. In this context, the effect of TS is far from being understood and the decision to start the therapy depends on a careful evaluation of the patient's characteristics.

Conflict of interest All authors certify that they have no actual or potential conflict of interest in relation to this article.

\section{References}

1. Corona G, Rastrelli G, Maggi M (2013) Diagnosis and treatment of late-onset hypogonadism: systematic review and meta-analysis of TRT outcomes. Best Pract Res Clin Endocrinol Metab 27(4):557-579

2. Huang G, Bhasin S, Tang ER et al (2013) Effect of testosterone administration on liver fat in older men with mobility limitation: results from a randomized controlled trial. J Gerontol A Biol Sci Med Sci 68(8):954-959

3. Tan WS, Low WY, Ng CJ et al (2013) Efficacy and safety of long-acting intramuscular testosterone undecanoate in aging men: a randomised controlled study. BJU Int 111(7):1130-1140
4. Caminiti G, Volterrani M, Iellamo F et al (2009) Effect of longacting testosterone treatment on functional exercise capacity, skeletal muscle performance, insulin resistance, and baroreflex sensitivity in elderly patients with chronic heart failure a doubleblind, placebo-controlled, randomized study. J Am Coll Cardiol 54(10):919-927

5. Malkin CJ, Pugh PJ, Jones RD, Kapoor D, Channer KS, Jones TH (2004) The effect of testosterone replacement on endogenous inflammatory cytokines and lipid profiles in hypogonadal men. J Clin Endocrinol Metab 89(7):3313-3318

6. Page ST, Amory JK, Bowman FD et al (2005) Exogenous testosterone $(\mathrm{T})$ alone or with finasteride increases physical performance, grip strength, and lean body mass in older men with low serum T. J Clin Endocrinol Metab 90(3):1502-1510

7. Tenover JS (1992) Effects of testosterone supplementation in the aging male. J Clin Endocrinol Metab 75(4):1092-1098

8. Francomano D, Bruzziches R, Barbaro G, Lenzi A, Aversa A (2014) Effects of testosterone undecanoate replacement and withdrawal on cardio-metabolic, hormonal and body composition outcomes in severely obese hypogonadal men: a pilot study. J Endocrinol Invest 37(4):401-411

9. Hackett G, Cole N, Bhartia M, Kennedy D, Raju J, Wilkinson P (2013) Testosterone replacement therapy with long-acting testosterone undecanoate improves sexual function and quality-oflife parameters vs. placebo in a population of men with type 2 diabetes. J Sex Med 10(6):1612-1627

10. Jones TH, Arver S, Behre HM et al (2011) Testosterone replacement in hypogonadal men with type 2 diabetes and/or metabolic syndrome (the TIMES2 study). Diabetes Care 34(4):828-837

11. Kalinchenko SY, Tishova YA, Mskhalaya GJ, Gooren LJ, Giltay EJ, Saad F (2010) Effects of testosterone supplementation on markers of the metabolic syndrome and inflammation in hypogonadal men with the metabolic syndrome: the double-blinded placebo-controlled Moscow study. Clin Endocrinol (Oxf) 73(5):602-612

12. Srinivas-Shankar U, Roberts SA, Connolly MJ et al (2010) Effects of testosterone on muscle strength, physical function, body composition, and quality of life in intermediate-frail and frail elderly men: a randomized, double-blind, placebo-controlled study. J Clin Endocrinol Metab 95(2):639-650

13. Kapoor D, Goodwin E, Channer KS, Jones TH (2006) Testosterone replacement therapy improves insulin resistance, glycaemic control, visceral adiposity and hypercholesterolaemia in hypogonadal men with type 2 diabetes. Eur $\mathrm{J}$ Endocrinol 154(6):899-906

14. Francomano D, Lenzi A, Aversa A (2014) Effects of five-year treatment with testosterone undecanoate on metabolic and hormonal parameters in ageing men with metabolic syndrome. Int $\mathbf{J}$ Endocrinol 2014:527470

15. Isidori AM, Lenzi A (2005) Risk factors for androgen decline in older males: lifestyle, chronic diseases and drugs. J Endocrinol Invest 28(3 Suppl):14-22

16. Ferlin A, Selice R, Carraro U, Foresta C (2013) Testicular function and bone metabolism-beyond testosterone. Nat Rev Endocrinol 9(9):548-554

17. Francis RM (1999) The effects of testosterone on osteoporosis in men. Clin Endocrinol (Oxf) 50(4):411-414

18. Aversa A, Bruzziches R, Francomano D et al (2012) Effects of long-acting testosterone undecanoate on bone mineral density in middle-aged men with late-onset hypogonadism and metabolic syndrome: results from a 36 months controlled study. Aging Male 15(2):96-102

19. Isidori AM, Giannetta E, Greco EA et al (2005) Effects of testosterone on body composition, bone metabolism and serum lipid 
profile in middle-aged men: a meta-analysis. Clin Endocrinol (Oxf) 63(3):280-293

20. Kenny AM, Kleppinger A, Annis K et al (2010) Effects of transdermal testosterone on bone and muscle in older men with low bioavailable testosterone levels, low bone mass, and physical frailty. J Am Geriatr Soc 58(6):1134-1143

21. Tracz MJ, Sideras K, Bolona ER et al (2006) Testosterone use in men and its effects on bone health. A systematic review and meta-analysis of randomized placebo-controlled trials. J Clin Endocrinol Metab 91(6):2011-2016

22. Tirabassi G, Delli MN, Gioia A, Biagioli A, Lenzi A, Balercia G (2014) Effects of testosterone replacement therapy on bone metabolism in male post-surgical hypogonadotropic hypogonadism: focus on the role of androgen receptor CAG polymorphism. J Endocrinol Invest 37(4):393-400

23. Amory JK, Watts NB, Easley KA et al (2004) Exogenous testosterone or testosterone with finasteride increases bone mineral density in older men with low serum testosterone. J Clin Endocrinol Metab 89(2):503-510

24. Svartberg J, Agledahl I, Figenschau Y, Sildnes T, Waterloo K, Jorde R (2008) Testosterone treatment in elderly men with subnormal testosterone levels improves body composition and BMD in the hip. Int J Impot Res 20(4):378-387

25. Corona G, Rastrelli G, Balercia G, Sforza A, Forti G, Maggi M (2012) Testosterone and cardiovascular risk in patients with erectile dysfunction. J Endocrinol Invest 35(9):809-816

26. Isidori AM, Giannetta E, Pozza C, Bonifacio V, Isidori A (2005) Androgens, cardiovascular disease and osteoporosis. J Endocrinol Invest 28(10 Suppl):73-79

27. Calof OM, Singh AB, Lee ML et al (2005) Adverse events associated with testosterone replacement in middle-aged and older men: a meta-analysis of randomized, placebo-controlled trials. J Gerontol A Biol Sci Med Sci 60(11):1451-1457

28. Fernandez-Balsells MM, Murad MH, Lane M et al (2010) Clinical review 1: adverse effects of testosterone therapy in adult men: a systematic review and meta-analysis. J Clin Endocrinol Metab 95(6):2560-2575

29. Haddad RM, Kennedy CC, Caples SM et al (2007) Testosterone and cardiovascular risk in men: a systematic review and metaanalysis of randomized placebo-controlled trials. Mayo Clin Proc 82(1):29-39

30. Xu L, Freeman G, Cowling BJ, Schooling CM (2013) Testosterone therapy and cardiovascular events among men: a systematic review and meta-analysis of placebo-controlled randomized trials. BMC Med 11:108

31. Corona G, Maseroli E, Rastrelli G et al (2014) Cardiovascular risk associated with testosterone boosting medications. Expert Opin Drug Safety (in press)

32. Ioannidis JP (2009) Adverse events in randomized trials: neglected, restricted, distorted, and silenced. Arch Intern Med 169(19):1737-1739

33. Huhtaniemi I, Forti G (2011) Male late-onset hypogonadism: pathogenesis, diagnosis and treatment. Nat Rev Urol 8(6):335-344

34. Radicioni AF, Ferlin A, Balercia G et al (2010) Consensus statement on diagnosis and clinical management of Klinefelter syndrome. J Endocrinol Invest 33(11):839-850

35. Wu FC, Tajar A, Beynon JM et al (2010) Identification of lateonset hypogonadism in middle-aged and elderly men. $\mathrm{N}$ Engl $\mathrm{J}$ Med 363(2):123-135

36. Isidori AM, Corona G, Aversa A et al (2014) The SIAMS-ED trial: a national, independent, multicentre study on cardiometabolic and hormonal impairment of men with erectile dysfunction treated with vardenafil. Int J Endocrinol 2014:858715

37. Bolona ER, Uraga MV, Haddad RM et al (2007) Testosterone use in men with sexual dysfunction: a systematic review and meta- analysis of randomized placebo-controlled trials. Mayo Clin Proc 82(1):20-28

38. Corona G, Isidori AM, Buvat $\mathbf{J}$ et al (2014) Testosterone supplementation and sexual function: a meta-analysis study. J Sex Med 11(6):1577-1592

39. Isidori AM, Buvat J, Corona G et al (2014) A critical analysis of the role of testosterone in erectile function: from pathophysiology to treatment-a systematic review. Eur Urol 65(1):99-112

40. Condorelli RA, Calogero AE, La VS (2013) Different profile of endothelial cell apoptosis in patients with Klinefelter's syndrome. J Endocrinol Invest 36(2):84-91

41. Jannini EA, Isidori AM, Aversa A, Lenzi A, Althof SE (2013) Which is first? The controversial issue of precedence in the treatment of male sexual dysfunctions. J Sex Med 10(10):2359-2369

42. Corona G, Rastrelli G, Vignozzi L, Mannucci E, Maggi M (2012) How to recognize late-onset hypogonadism in men with sexual dysfunction. Asian J Androl 14(2):251-259

43. Johnson JM, Nachtigall LB, Stern TA (2013) The effect of testosterone levels on mood in men: a review. Psychosomatics 54(6):509-514

44. Amanatkar HR, Chibnall JT, Seo BW, Manepalli JN, Grossberg GT (2014) Impact of exogenous testosterone on mood: a systematic review and meta-analysis of randomized placebo-controlled trials. Ann Clin Psychiatry 26(1):19-32

45. Cotter AG, Powderly WG (2011) Endocrine complications of human immunodeficiency virus infection: hypogonadism, bone disease and tenofovir-related toxicity. Best Pract Res Clin Endocrinol Metab 25(3):501-515

46. Rochira V, Zirilli L, Orlando G et al (2011) Premature decline of serum total testosterone in HIV-infected men in the HAART-era. PLoS ONE 6(12):e28512

47. Bhasin S, Cunningham GR, Hayes FJ et al (2010) Testosterone therapy in men with androgen deficiency syndromes: an Endocrine Society clinical practice guideline. J Clin Endocrinol Metab 95(6):2536-2559

48. Buvat J, Maggi M, Guay A, Torres LO (2013) Testosterone deficiency in men: systematic review and standard operating procedures for diagnosis and treatment. J Sex Med 10(1):245-284

49. Corona G, Baldi E, Maggi M (2011) Androgen regulation of prostate cancer: where are we now? J Endocrinol Invest 34(3):232-243

50. Marks LS, Mazer NA, Mostaghel E et al (2006) Effect of testosterone replacement therapy on prostate tissue in men with lateonset hypogonadism: a randomized controlled trial. JAMA 296(19):2351-2361

51. Merza Z, Blumsohn A, Mah PM et al (2006) Double-blind placebo-controlled study of testosterone patch therapy on bone turnover in men with borderline hypogonadism. Int $\mathbf{J}$ Androl 29(3):381-391

52. Snyder PJ, Peachey H, Hannoush P et al (1999) Effect of testosterone treatment on bone mineral density in men over 65 years of age. J Clin Endocrinol Metab 84(6):1966-1972

53. Steidle C, Schwartz S, Jacoby K, Sebree T, Smith T, Bachand R (2003) AA2500 testosterone gel normalizes androgen levels in aging males with improvements in body composition and sexual function. J Clin Endocrinol Metab 88(6):2673-2681

54. Aversa A, Bruzziches R, Francomano D et al (2010) Effects of testosterone undecanoate on cardiovascular risk factors and atherosclerosis in middle-aged men with late-onset hypogonadism and metabolic syndrome: results from a 24 month, randomized, doubleblind, placebo-controlled study. J Sex Med 7(10):3495-3503

55. Cavallini G, Caracciolo S, Vitali G, Modenini F, Biagiotti G (2004) Carnitine versus androgen administration in the treatment of sexual dysfunction, depressed mood, and fatigue associated with male aging. Urology 63(4):641-646 
56. Shahidi NT (1973) Androgens and erythropoiesis. N Engl J Med 289(2):72-80

57. Drinka PJ, Jochen AL, Cuisinier M, Bloom R, Rudman I, Rudman D (1995) Polycythemia as a complication of testosterone replacement therapy in nursing home men with low testosterone levels. J Am Geriatr Soc 43(8):899-901

58. Hajjar RR, Kaiser FE, Morley JE (1997) Outcomes of long-term testosterone replacement in older hypogonadal males: a retrospective analysis. J Clin Endocrinol Metab 82(11):3793-3796

59. Morley JE, Perry HM III, Kaiser FE et al (1993) Effects of testosterone replacement therapy in old hypogonadal males: a preliminary study. J Am Geriatr Soc 41(2):149-152

60. Liu PY, Swerdloff RS, Christenson PD, Handelsman DJ, Wang C (2006) Rate, extent, and modifiers of spermatogenic recovery after hormonal male contraception: an integrated analysis. Lancet 367(9520):1412-1420

61. Matsumoto AM, Sandblom RE, Schoene RB et al (1985) Testosterone replacement in hypogonadal men: effects on obstructive sleep apnoea, respiratory drives, and sleep. Clin Endocrinol (Oxf) 22(6):713-721

62. Snyder PJ, Peachey H, Hannoush P et al (1999) Effect of testosterone treatment on body composition and muscle strength in men over 65 years of age. J Clin Endocrinol Metab 84(8):2647-2653

63. Rolf C, Knie U, Lemmnitz G, Nieschlag E (2002) Interpersonal testosterone transfer after topical application of a newly developed testosterone gel preparation. Clin Endocrinol (Oxf) 56(5):637-641 